\title{
Validation of a multivariable Relay-Based PID Autotuner with Specified Robustness
}

\author{
Robin De Keyser, Anca Maxim, Cosmin Copot, Clara M. Ionescu \\ Ghent University \\ Department of Electrical energy, Systems and Automation \\ Technologiepark 913, 9052 Gent, Belgium \\ \{Robain.DeKeyser, Anca.Maxim, Cosmin.Copot, ClaraMihaela.Ionescu\}@UGent.be
}

\begin{abstract}
This paper presents a multivariable relay-based PID autotuning strategy, which ensures a specified modulus margin (i.e. robustness). The algorithm is applied on the coupled quadruple tanks from Quanser. The system is challenging for control since it presents non-minimum phase transmission zeros. The performance of the autotuner is validated against a computer-aided design tool based on the frequency response, i.e. FRTool. The experimental results suggest that the proposed autotuning procedure has similar performance as the control design based on full knowledge of the system. This is a remarkable conclusion and provides a good motivation to claim that our algorithm may be useful in chemical process applications where full knowledge of the systems model is still a burden for the control engineer.
\end{abstract}

\section{Introduction}

Industrial applications of inter-connected systems are manifold and present difficult control issues, such as time delays, multivariable interaction, non-minimum phase dynamics, etc [1]. Process identification is usually very challenging and time consuming. To simplify this task and to reduce the time required for it, many PID regulators nowadays include autotuning capabilities, i.e. they are equipped with a mechanism capable of computing the correct parameters automatically when the regulator is connected to the plant. A specific class of autotuners use relay feedback in order to obtain some information on the process frequency response [2,3]. For multivariable systems, the autotuning procedure needs to take into account the cross-coupling dynamics in order to converge to stable closed loop controllers [4]. Using a relay controller, the outputs will oscillate in the form of limit cycles (after an initial transient). The controller parameters are then iteratively obtained such that these output oscillations converge to the critical frequency of the entire coupled system $[4,5]$. The number of iterations is usually related to the number of input-output pairings [6].

Classical PID autotuning approaches such as the Astrom-Hagglund (AH) autotuner and Phase Margin (PM) autotuner [7, 8, 9, 10], identify the critical point on the process frequency response using such relay feedback. Their advantage is that they are very simple to apply, i.e. few choices are left for the user (which is indeed an advantage if the industrial user is lacking theoretical control engineering insight). The autotuner performance is compared against model-based techniques, employing a computer-aided design toolbox based on the frequency response (i.e. FRTool, also developed in-house) [11]. A typical chemical process setup for laboratory use is the quadruple tank from Quanser (www.Quanser.com). The process is multivariable, highly coupled and presents nonminimum phase transmission zero. This setup is then used to illustrate the effectiveness of the proposed algorithm in real-life tests.

The outline of this paper is as follows. The setup is briefly given in section 2 . The system model and identification (for validation purposes only) are presented in the third section. The control algorithms are described in the fourth section and their results are presented in the fifth section. A conclusion section summarizes the main outcome of this work.

\section{Description of the MIMO system}

Figure 1 presents the schematic overview of the multivariable system represented by the quadruple tank water level control. The control objective is to regulate the level of the water in the tanks below by manipulating the water flows. The plant has two manipulated inputs, i.e. the voltages of the two pumps $V_{p 1}(t)$ and $V_{p 2}(t)$ (expressed in Volts), and two controlled outputs, i.e. the water levels of the two tanks below, $L_{2}(t)$, the level in Tank2, and $L_{4}(t)$, the level in Tank4 (both expressed in $\mathrm{cm}$ ). There is a strong coupling effect between the inputs and the outputs, (e.g. in Tank2 there are two inputs: the flow from Pump1 through Out2, marked with dashed red line, and the flow from Pump2 through Out1, denoted with green continuous 
line, that is the output flow from Tank1). Hence, the controlled level in Tank 2 is influenced by the two inputs, and by adjusting the percentage of water flow from each input, one can change the system for having minimum phase or non-minimum phase dynamics.

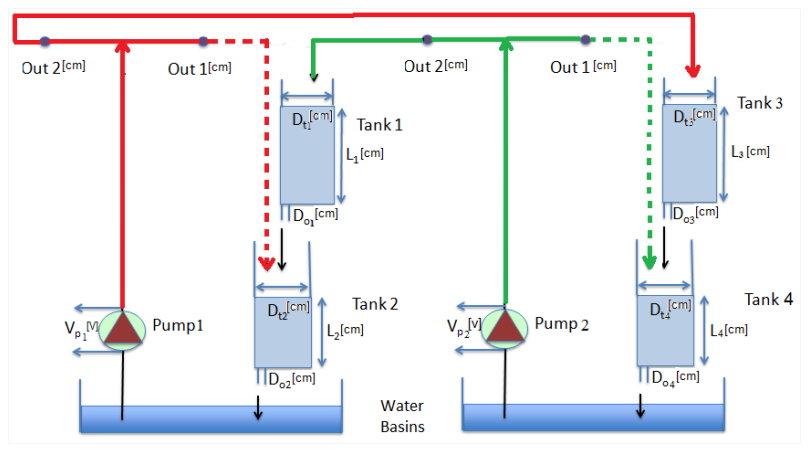

Figure 1. Schematic diagram of the quadruple tank process from Quanser

Based on the configuration depicted in Figure 1, in Tank2 there is a greater flow coming from Pump2, via Tank1, than the flow coming directly from Pump1. This exotic situation originates from the fact that the outlet diameter Out1 is bigger than the diameter Out2, while the outgoing orifices from each tank $D_{o_{i}}, \mathrm{i}=1 \ldots 4$ have all the same diameter. The same situation applies for Tank4. It follows the conclusion that the dominant flow in the tanks 2 and 4 comes from the manner in which the physical coupling is implemented via the choice of the setup.

\section{Modelling and identification}

In order to simplify matters, the modelling principle for a single-input single output setup is given below, but it is valid for the multivariable setup as well. The outflow from Tank1 can be expressed as $F_{o 1}=A_{o 1} v_{o 1}$, with $v_{o 1}$ the flow velocity. The cross-section area of the outflow orifice in Tank1 can be expressed as $A_{o 1}=\frac{1}{4} \pi D_{o 1}^{2}$, with $D_{o 1}$ the diameter. Using Bernoullis equation, we have that:

$$
F_{o 1}=A_{o 1} \sqrt{2} \sqrt{g L_{1}}
$$

Using the mass balance equation in Tank1 it follows that

$$
A_{t 1} \frac{d}{d t} L_{1}=F_{i 1}-F_{o 1}
$$

Substituting for $F_{i 1}$ and $F_{o 1}$ we have that:

$$
\frac{d}{d t} L_{1}=\frac{K_{f} V_{p}-A_{o 1} \sqrt{2} \sqrt{g L_{1}}}{A_{t 1}} .
$$

In steady-state, all time derivatives are zero and it results $K_{f} V_{p 0}-A_{o 1} \sqrt{2} \sqrt{g L_{10}}=0$. For any value of the desired level in Tank1 $\left(L_{10}=10 \mathrm{~cm}\right)$, the value of the pump voltage in equilibrium can be calculated. Applying linearization and Laplace transform, we have that:

$$
\frac{L_{11}}{V_{p}}(s)=\frac{K_{1}}{\tau_{1} s+1},
$$

where $K_{1}=\frac{K_{f} \sqrt{2} \sqrt{g L_{10}}}{A_{o 1} g}$ and $\tau_{1}=\frac{A_{t 1} \sqrt{2} \sqrt{g L_{10}}}{A_{o 1} g}$ Similarly, the outflow from Tank2 can be expressed as $F_{o 2}=$ $A_{o 2} v_{o 2}$. The cross-section area of the outflow orifice in Tank2 can be expressed as $A_{o 2}=\frac{1}{4} \pi D_{o 2}^{2}$. Using Bernoullis equation and the mass balance equation in Tank2 it follows that:

$$
A_{t 2}\left(\frac{d}{d t} L_{2}\right)=F_{i 2}-F_{o 2}
$$

Substituting for $F_{i 2}$ and $F_{o 2}$ assuming steady-state, it follows that:

$$
L_{10}=\frac{A_{o 2}^{2} L_{20}}{A_{o 1}^{2}} .
$$

Applying linearization and Laplace transform, we have that:

$$
\frac{L_{21}}{L_{11}}(s)=\frac{K_{2}}{\tau_{2} s+1},
$$

where $K_{2}=\frac{A_{o 1} \sqrt{L_{20}}}{A_{o 2} \sqrt{L_{10}}}$ and $\tau_{2}=\frac{A_{t 2} \sqrt{2} \sqrt{g L_{20}}}{A_{o 2} g}$

Identification was performed in order to allow validation of our autotuner method. A PRBS signal was applied to the plant around the operating point of $10 \mathrm{~cm}$. In Figure 2 are shown the deviation values of the two outputs, where 0 corresponds with the operating point of $10 \mathrm{~cm}$. Signals were sampled at a period of 1 second and de-trended. Prediction error estimation algorithm has been used, resulting in the following transfer function matrix:

$$
G(s)=\left[\begin{array}{cc}
\frac{1.78}{22.71 s+1} & \frac{2.49}{178.8 s^{2}+26.74 s+1} \\
\frac{2.76}{159.2 s^{2}+33.41 s+1} & \frac{1.28}{15.92 s+1}
\end{array}\right]
$$

The transmission zeros are:

$$
\begin{aligned}
& z_{1}=-0.32 ; z_{2}=0.07 \\
& z_{3}=-0.06 ; z_{4}=-0.04
\end{aligned}
$$

To check input-output pairings, we apply the relative gain array (RGA) method and obtain:

$$
\Lambda=\left[\begin{array}{cc}
-0.49 & 1.49 \\
1.49 & -0.49
\end{array}\right]
$$

The RGA matrix $\Lambda$ suggests that the pairing 1-1/2-2 is not suitable, since the main diagonal has negative values. This outcome was expected, as already mentioned in the description of the process, since $u_{2}$ is the dominant input for $y_{1}$. Hence, the 1-2/2-1 pairing from figure 3 will be further used in the remaining of the paper. In this schematic representation of the MIMO closed loops, $w_{1,2}$ denote the setpoints for the outputs $y_{1,2}, C_{1,2}$ are the controllers for each loop, and $G_{i, j}$ with $i, j=1 \ldots 2$ are the transfer functions from input $j$ to the output $i$. 

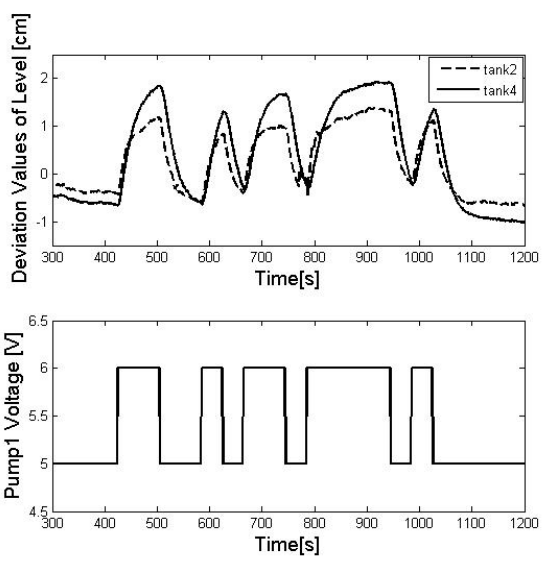

Figure 2. The PRBS signal and the output levels in the two lower tanks

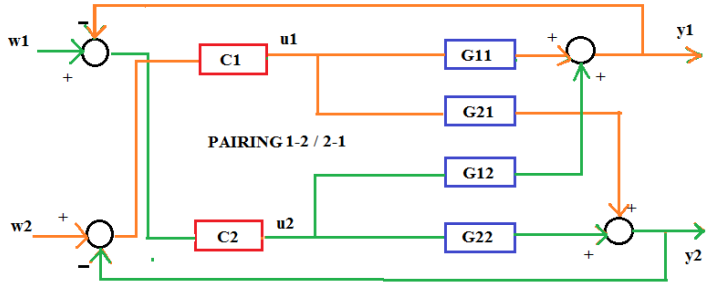

Figure 3. Control Scheme with 1-2/2-1 pairing

\section{Control strategies}

\subsection{Proposed autotuning algorithm}

The proposed autotuner is based on the classic idea of exposing the process to an experiment, during the normal operation. This requires careful design of the excitation signal, to avoid de-stabilizing the process from its operating point. Traditional relay-based autotuning methods such as AH identify one point on the Nyquist curve of the process P: the intersection of the process beeline with the negative real axis, ref. Figure 4 [8].

Using an appropriate PID controller, denoted by $\mathrm{C}$, this point is then moved to a specific point in the complex Nyquist plane; e.g. for the original AH-tuner, the beeline of $C * P$ goes through the specific point $-0.6-0.28 j$ (distance to the point -1 is then 0.5 ). The insights from [10] show that specification of only 1 point in the Nyquist plane might be sufficient for some type of processes, but might as well result in poor (low) modulus margin for other types of processes.

The development of the proposed MIMO autotuning algorithm can be described in an iteratively manner.

Step1: Simultaneously close all the loops with a relay feedback in order to take into account the coupling effects while tuning the controller parameters $[6,4]$. As a result, both outputs will oscillate at the common critical

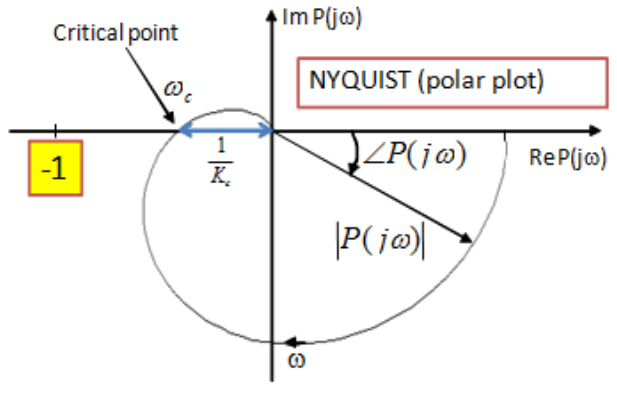

Figure 4. The Nyquist plot of the process and its intersection with the negative real axis

frequency of the MIMO system (i.e. the critical frequency of the dominant loop).

Step2: Arbitrarily chose one output and find the oscillations magnitude and phase.

Step3: Add a delay to the 'the chosen' output, while maintaining the same relay on the other loop (as in Step1). Find the oscillations magnitude and phase for the relay+delay loop.

Step4: Compute a PID controller for 'the chosen' loop.

Step5: Replace the relay+delay from the loop selected at step 2 with the computed controller (Step 4). Find for the second loop the oscillations magnitude and phase.

Step6: Repeat steps 3-4 (with the mention that the other loop is closed with PID).

Step7: Alternate between the two loops and repeat steps 5-6 for each loop until the output magnitude and phase found at Step 3 does not change from those found at Step 2.

Thus, the convergence of the algorithm is established when the output magnitude and phase in the relay+delay test is the same like in the relay test. This was performed with the help of the designer, but can be easily automated. A condition for convergence is that minimum one of the loops in the system has a characteristic locus with at least 180 degrees phase lag $[4,5]$.

The controllers from this paper were obtained after four iterations of the above procedure. Next, the methodology behind steps 2-4 is presented. The development of the proposed autotuning algorithm is based on imposing a user-specified robustness. The robustness specification can be translated using Nyquist plots as a circle of specified radius (r) around the point -1 as drawn in Figure 5 ( $r=$ the Modulus Margin, $0<r<1$ ).

Using a relay test, two points from the process frequency response and critical frequency are obtained.

$$
P\left(j \omega_{c}\right)=M_{P} e^{j \varphi_{P}}=M_{P} e^{j 180^{\circ}}=-M_{P}
$$

The algorithm searches for the angle $\alpha$ under which the Nyquist curve of the process and controller $P(j \omega) C(j \omega)$ 


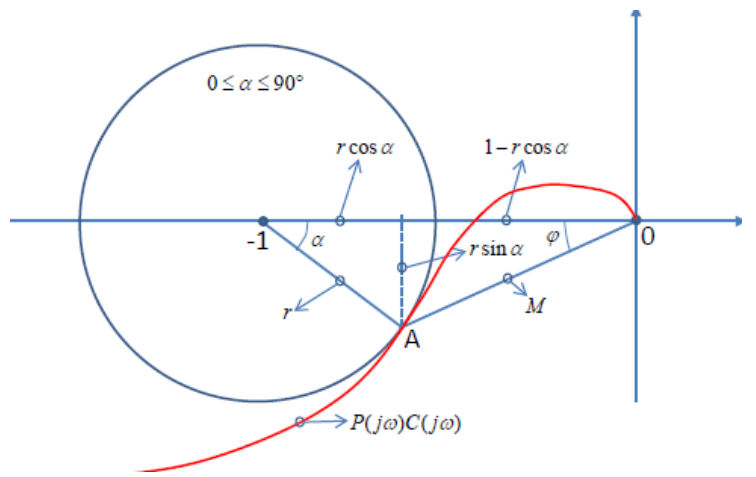

Figure 5. Supporting figure for algorithm development

touches the circle in $A$. From (11) we get:

$$
\begin{aligned}
& P\left(j \omega_{c}\right) C\left(j \omega_{c}\right)=M_{P} e^{j \varphi_{P}} * M_{C} e^{j \varphi_{C}}= \\
& \quad=M_{P} M_{C} e^{j\left(180^{\circ}+\varphi_{C}\right)}=M e^{j\left(180^{\circ}+\varphi\right)}
\end{aligned}
$$

where:

$$
\varphi=\varphi_{C} \text { and } M=M_{P} M_{C}
$$

Next, the textbook frequency response for the PID controller is written:

$$
\begin{array}{r}
C\left(j \omega_{c}\right)=K_{p}\left(1+\frac{1}{T_{i} j \omega_{c}}+T_{d} j \omega_{c}\right)= \\
K_{p}\left[1+j\left(T_{d} \omega_{c}-\frac{1}{T_{i} \omega_{c}}\right)\right]
\end{array}
$$

\section{Considering}

$$
\tan \varphi_{C}=T_{d} \omega_{c}-\frac{1}{T_{i} \omega_{c}}
$$

and

$$
M_{C}=K_{p} \sqrt{1+\tan \varphi_{C}^{2}}=\frac{K_{p}}{\cos \varphi_{C}}
$$

and imposing two identical zeros for the controller $\left(T_{i}=\right.$ $4 T_{d}$ ), we obtain

$$
\tan \varphi_{C}=T_{d} \omega_{c}-\frac{1}{4 T_{d} \omega_{c}} \Rightarrow T_{d} \omega_{c}=\frac{1+\sin \varphi}{2 \cos \varphi}
$$

Replacing (16) and (17) into (13) we get the parameters for the PID controller:

$$
\begin{aligned}
& K_{p}=\frac{M}{M_{P}} \cos \varphi \\
& T_{d}=\frac{1+\sin \varphi}{2 \omega_{c} \cos \varphi} \\
& T_{i}=4 T_{d}
\end{aligned}
$$

where $M$ and $\varphi$ are obtained from Pythagorean Theorem applied in figure 5:

$$
\begin{gathered}
M=\sqrt{(r \sin \alpha)^{2}+(1-r \cos \alpha)^{2}}= \\
=\sqrt{r^{2}-2 r \cos \alpha+1} \\
\varphi=\arctan \frac{r \sin \alpha}{1-r \cos \alpha}
\end{gathered}
$$

Now we can express the variation of the modulus with the phase, which describes the tangent to a circle of radius $\mathrm{r}$ around the point -1 :

$$
\left.\frac{d M}{d \varphi}\right|_{\omega_{c}}=\frac{\mathrm{dM} / \mathrm{d} \omega}{\mathrm{d} \varphi / \mathrm{d} \omega}
$$

with

$$
\frac{d M}{d \omega}=\frac{d\left(M_{P} M_{C}\right)}{d \omega}=M_{P} \frac{d M_{C}}{d \omega}+\frac{M}{M_{P}} \frac{d M_{P}}{d \omega}
$$

and

$$
\frac{d \varphi}{d \omega}=\frac{d\left(\varphi_{P}+\varphi_{C}\right)}{d \omega}=\frac{d \varphi_{P}}{d \omega}+\frac{d \varphi_{C}}{d \omega}
$$

In order to compute, we need

$$
\frac{d M_{C}}{d \omega}=\frac{d M_{C}}{d \tan \varphi_{C}} \frac{d \tan \varphi_{C}}{d \omega}
$$

which is further equal to

$$
K_{p} \sin \varphi_{C} \frac{1}{\omega \cos \varphi_{C}}=\frac{M \sin \varphi_{C}}{M_{P} \omega}
$$

and

$$
\frac{d \varphi_{C}}{d \omega}=\frac{d \tan \varphi_{C} / d \omega}{d \tan \varphi_{C} / d \varphi_{C}}=\frac{1 / \omega \cos \varphi_{C}}{1 / \cos \varphi_{C}{ }^{2}}=\frac{\cos \varphi_{C}}{\omega}
$$

Substituting, we have

$$
\left.\frac{d \mathrm{M}}{d \varphi}\right|_{\omega_{C}}=\mathrm{M} \frac{\frac{\sin \varphi}{\omega_{\mathrm{c}}}+\left.\frac{1}{\mathrm{M}_{\mathrm{P}}} \frac{d M_{P}}{d \omega}\right|_{\omega_{c}}}{\frac{\cos \varphi}{\omega_{\mathrm{c}}}+\left.\frac{\mathrm{d} \varphi_{\mathrm{P}}}{\mathrm{d} \omega}\right|_{\omega_{c}}}
$$

Since for autotuning purposes we do not have a model for the process, we will approximate the derivative of the process frequency response in $\omega_{C}$ using differences:

$$
\begin{gathered}
\left.\left.\frac{d M_{P}}{d \omega}\right|_{\omega_{c}} \approx \frac{\Delta M_{P}}{\Delta \omega}\right|_{\omega_{c}}=\frac{M_{P}\left(j \omega_{c}\right)-M_{P}\left(j \omega^{\prime}\right)}{\omega_{c}-\omega^{\prime}} \\
\left.\left.\frac{d \varphi_{P}}{d \omega}\right|_{\omega_{c}} \approx \frac{\Delta \varphi_{P}}{\Delta \omega}\right|_{\omega_{c}}=\frac{\varphi_{P}\left(j \omega_{c}\right)-\varphi_{P}\left(j \omega^{\prime}\right)}{\omega_{c}-\omega^{\prime}}
\end{gathered}
$$

where $M_{P}\left(j \omega^{\prime}\right)$ and $\varphi_{P}\left(j \omega^{\prime}\right)$ are the modulus and phase of the process at a frequency $\omega^{\prime}$ which is close to the critical frequency $\omega_{c}$. This can be easily obtained using a relay test with time delay, $\tau_{d}=\frac{\Delta \varphi_{P}}{\omega_{C}}$, corresponding to a specified $\Delta \varphi_{P}$ [12]. Next, we compute the variation of the modulus with the phase, in the point $A$, from figure 5:

$$
\left.\frac{d M}{d \varphi}\right|_{\mathrm{A}}=\frac{\mathrm{dM} / \mathrm{d} \alpha}{\mathrm{d} \varphi / \mathrm{d} \alpha}=\mathrm{M} \frac{\sin \alpha}{\cos \alpha-\mathrm{r}}
$$

where

$$
\begin{gathered}
\frac{d M}{d \alpha}=\frac{r \sin \alpha}{M} \\
\frac{d \varphi}{d \alpha}=\frac{r(\cos \alpha-r)}{M^{2}}
\end{gathered}
$$


Then, by finding iteratively the angle $\alpha^{*}$ for which the error $\left|\frac{d M}{d \varphi}\right|_{\omega_{C}}-\left.\frac{d M}{d \varphi}\right|_{A} \mid$ is minimum, we obtain the optimal parameters of the controller for a specified modulus margin $r$.

The procedure presented above, can be summarized as:

1) Find $\omega_{c}$ and $M_{P}\left(\omega_{c}\right)$ via relay test $\left[\varphi_{c}=-180^{\circ}\right]$

2) Find $\omega_{c}^{\prime}, M_{P}\left(\omega_{c}^{\prime}\right)$ and $\varphi_{P}\left(\omega_{c}^{\prime}\right)$ via a relay+delay test

3) Calculate $\frac{\Delta M_{P}}{\Delta \omega}$ and $\frac{\Delta \varphi_{P}}{\Delta \omega}$

4) For $\alpha=0 \ldots 90^{\circ}$, calculate $M(\alpha), \varphi(\alpha)$ and $\delta(\alpha)=\left|\frac{d M}{d \varphi}\right|_{\omega_{C}}-\left.\frac{d M}{d \varphi}\right|_{A} \mid$

5) Calculate $M\left(\alpha^{*}\right)$ and $\varphi\left(\alpha^{*}\right)$ with $\alpha^{*}=\arg \min _{\alpha} \delta(\alpha)$

6) Calculate $\left\{K_{p}, T_{i}, T_{d}\right\}$.

This method of autotuning has been successfully validated on numerous examples for single input-single output systems $[12,13]$.

\subsection{Computer Aided PID Design: FRTool}

In this paper, a proof of concept for a MIMO autotunner is shown, thus as a reference performance the autotuner is compared against an 'in-house' developed tool, namely the Frequency Response tool (FRtool) for Matlab as described in [11]. The next step could be comparing the performance against a controller tuned for MIMO processes [14]. Figure 6 shows the tuning of the PID controller on the process $P(s)$ with the specifications: overshoot $\% O S<5 \%$, robustness $R o>0.7$ and settling time $T_{s}<200$ seconds. In this figure, $C$ denotes controller and $P$ denotes process transfer function (in this case, the closed loop transfer function, from input 1 to output 1 , taking into account the coupling effect). Also in the figure, with the blue continuous line is denoted the Nichols plot corresponding with the first loop and the numerical values of the gain and zeros are given, while with black dashed line, the interval for all MIMO loops is marked. The corresponding PID parameters are given in Table 1. Notice that $\mathrm{P}(\mathrm{s})$ in case of multivariable system is the total closed loop of the loop(s), which are not employed in this tuning step. In this way, interaction is already taken into account. A simple $K=1$ controller was put on the other loops, to be able to close the loop.

\section{Results and Conclusions}

Tuning of the model-based controllers via FRTool has been done using the specifications described in section 4.2. The FRTOOL-PID controller parameters are given in table 1. Tuning of the MM controller has been performed using a relay test on the real plant for each loop, giving a robustness specification of 0.7 (70\%). An example of such a relay feedback test on the real plant is given in Figure 7. The final MM-PID controller parameters are also given in table 1.

Figure 8 depicts the results for setpoint tracking closed loop performance for the PID controllers tuned with FR-

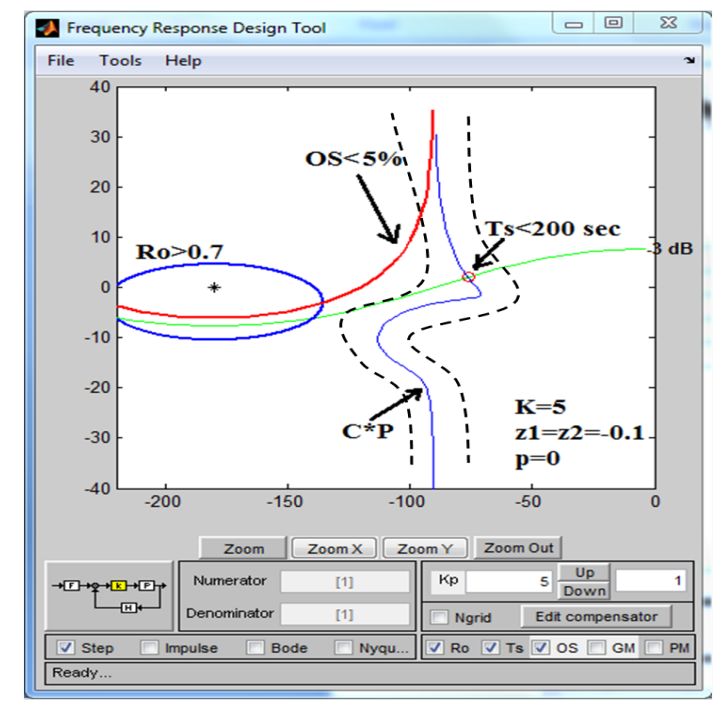

Figure 6. The Nichols plot of the FRTool CAD interactive graphical user interface.

Tool and with MM autotuner. The controllers for Tank2 and Tank4 are denoted by FR2, MM2 and FR4, MM4, respectively. The reference for water level in Tank2 is changed from $10 \mathrm{~cm}$ to $11 \mathrm{~cm}$, while keeping the reference for water level in Tank4 constant to $10 \mathrm{~cm}$. Next, the reference for Tank 4 as been changed to $11 \mathrm{~cm}$, while keeping the water level in Tank 2 constant to $11 \mathrm{~cm}$. Coupling effects are observed, but remarkably, both controllers (i.e. the model based PID and the PID autotuner) have similar closed loop performance.

Figure 9 depicts the results for disturbance rejection performance for the same PID controllers. The disturbance consists in eliminating the water from tank 1 directly in the reservoir, without going to Tank2 first. As a result, the level in Tank2 drops and the controller changes the pump voltage to recover the reference value of $11 \mathrm{~cm}$. Due to the coupling between the pumps (recall figures 1), the level in Tank4 will be also disturbed. Again, both model based PID controllers and PID autotuners have similar performance.

\section{Table 1. Controller parameters. See text for explanation.}

\begin{tabular}{cccc}
\hline & $K_{p}$ & $T_{i}$ & $T_{d}$ \\
\hline \hline$F R 2$ & 1 & 5 & 20 \\
$F R 4$ & 1.1 & 4.54 & 18.18 \\
$M M 2$ & 0.86 & 4.96 & 19.16 \\
$M M 4$ & 1.09 & 5.19 & 20.39 \\
\hline
\end{tabular}

This paper presented successful real-life tests of a novel PID autotuning procedure based on relay feedback for multivariable system with transmission zero and significant coupling. Further extension of this work could be the validation on other MIMO processes. 

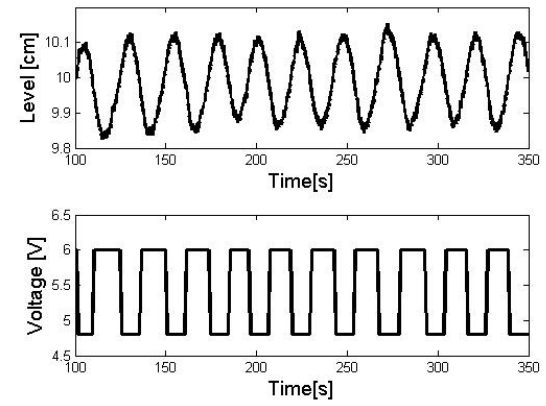

Figure 7. Example of relay test on the real plant
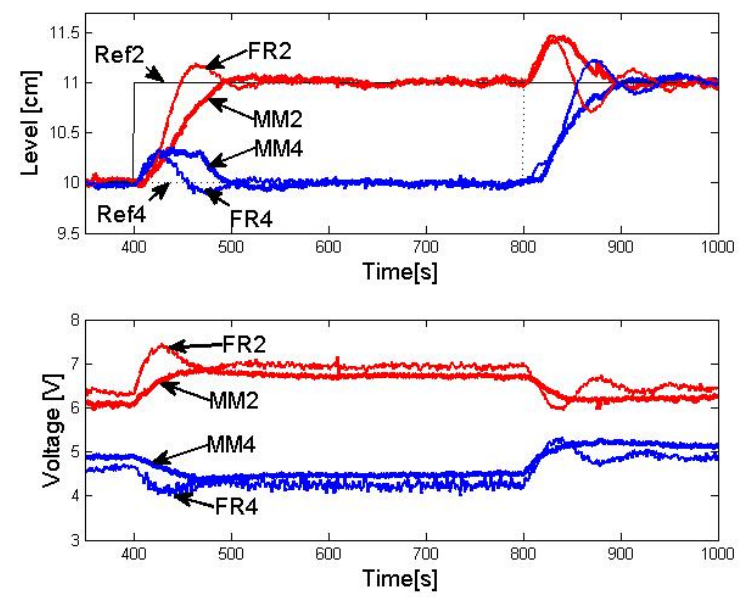

Figure 8. Real life test for setpoint tracking

\section{References}

[1] B.W. Bequette, Process Control: Modelling, Design and Simulation. Pretice-Hall, NJ, 2003.

[2] C.C. Yu, Autotuning of PID controllers - A relay feedback approach. Springer-Verlag, London, 2006.

[3] R. De Keyser, C.M. Ionescu, A comparative study of three relay-based PID autotuners, in Proc. of the IASTED Conf. On Modelling, Identification and Control (AsiaMIC 2010), Phuket, Thailand, pp 303310, 2010.

[4] Q-G. Wang, B. Zou, T-H. Lee, Q. Bi, Auto-tuning of multivariable PID controllers from decentralized relay feedback, Automatica, 33(3), pp. 319-330,1997.

[5] Q-C. Wang, Z. Ye, W-J. Cai, C-C. Hang, PID Control for Multivariable Processes, SpringerVerlag Berlin Heidelberg. 2008

[6] M. Johnson, M. Moradi, PID Control (Chapters 511), Springer-Verlag Ltd. London, 2005
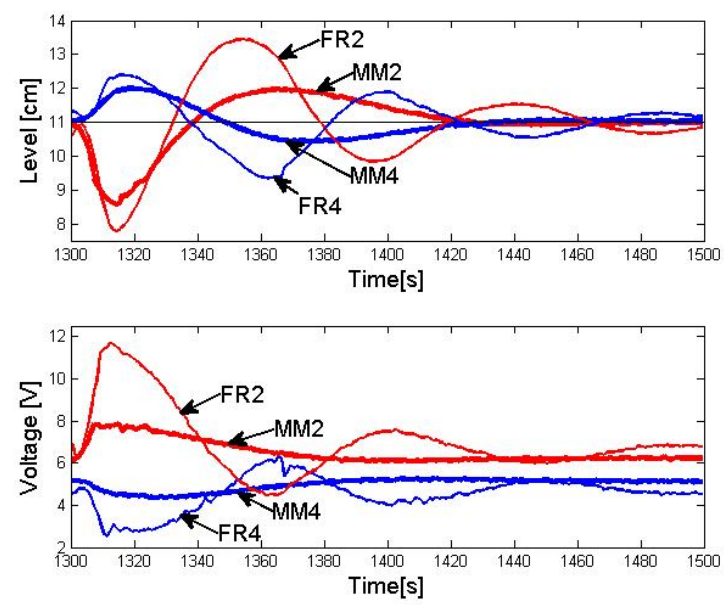

Figure 9. Real life test for disturbance rejection

[7] K.J. Astrom, C.C. Hang, P. Persson, W.K. Ho, Towards intelligent PID control, Automatica 28, pp. $1-9,1992$.

[8] K.J. Astrom, T. Hagglund, Advanced PID Control, ISA, 2006.

[9] C.C. Hang, K.J. Astrom, W.K. Ho, Refinements of the Ziegler-Nichols tuning formula, IEEE Proc. Design, Control Theory and Appl. 138, pp. 111118,1991 .

[10] C.M. Ionescu, R. De Keyser, The next generation of relay-based PID autotuners (PART1): Some insights on the performance of simple relay-based PID autotuners, IFAC Conf. on Advances in PID Control (PID12), Brescia Italy, pp. 122-127, 2012.

[11] R. De Keyser, C.M. Ionescu, FRtool: a frequency response tool for CACSD in Matlab, IEEE Conf. on Computer Aided Control Systems Design, Munich, pp. 2275-2280, 2006.

[12] R. De Keyser, O. Joita, C.M. Ionescu, The next generation of relay-based PID autotuners (PART 2): A simple relay based PID autotuner with specified modulus margin, IFAC Conf. on Advances in PID Control, Brescia, Italy, pp. 128-133, 2012.

[13] O. Joita, R. De Keyser, Validation of a simple relay based PID autotuner with specified modulus margin, IEEE Int. Conf. on Automation, Quality and Testing, Robotics, Romania, DOI 978-1-4673-0703-1/12, 6 pages , 2012.

[14] F.Vazquez, F. Morilla, S. Dormido, An iterative method for tuning decentralized PID controllers, Proc. of the 14th World Congress of the IFAC. Beijing, China, 1999. 